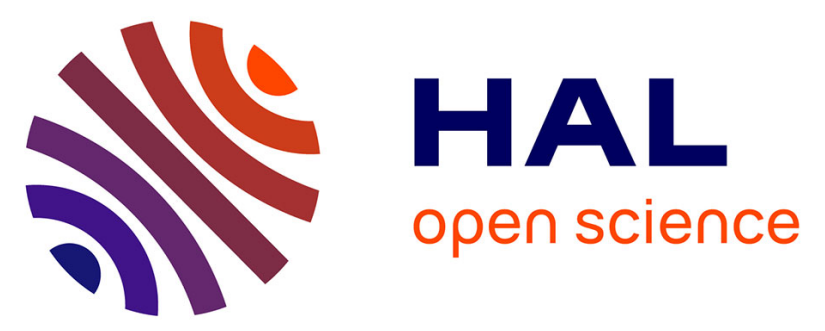

\title{
Données nouvelles sur la morphogenèse et les paléo-environnements tardiglaciaires et holocènes dans la vallée de l'oued Chéria-Mezeraa (Nemencha, Algérie orientale)
}

\author{
Jean-Louis Ballais, Mohamed Tahar Benazzouz
}

\section{To cite this version:}

Jean-Louis Ballais, Mohamed Tahar Benazzouz. Données nouvelles sur la morphogenèse et les paléoenvironnements tardiglaciaires et holocènes dans la vallée de l'oued Chéria-Mezeraa (Nemencha, Algérie orientale). Méditerranée: revue géographique des pays méditerranéens, 1994, Géographie physique de l'Algérie orientale, 80 (3-4), pp.59-71. 10.3406/medit.1994.2859 . hal-01564810

\section{HAL Id: hal-01564810 \\ https://hal-amu.archives-ouvertes.fr/hal-01564810}

Submitted on 26 Jul 2017

HAL is a multi-disciplinary open access archive for the deposit and dissemination of scientific research documents, whether they are published or not. The documents may come from teaching and research institutions in France or abroad, or from public or private research centers.
L'archive ouverte pluridisciplinaire HAL, est destinée au dépôt et à la diffusion de documents scientifiques de niveau recherche, publiés ou non, émanant des établissements d'enseignement et de recherche français ou étrangers, des laboratoires publics ou privés. 


\section{Données nouvelles sur la morphogenèse et les paléo- environnements tardiglaciaires et holocènes dans la vallée de l'oued Chéria-Mezeraa (Nemencha, Algérie orientale)}

In: Méditerranée, Tome 80, 3-4-1994. Géographie physique de l'Algérie orientale. pp. 59-71.

\section{Résumé}

L'étude de l'évolution de la vallée de l'oued Chéria-Mezeraa confirme l'existence d'une morphogenèse tardiglaciaire caractérisée par l'accumulation de cônes alluviaux. L'Holocène moyen et supérieur est bien représenté et permet la mise en évidence d'une crise morphogénique entre environ 4000 et 2400 B.P., et d'une période à bilan hydrique plus favorable que l'actuel vers $2400-2$ 200 B.P. L'Holocène se caractérise également par l'existence de trois phases d'entaille des cours d'eau mais elles n'individualisent que deux terrasses.

\section{Abstract}

The study of the evolution of the wadi Chéria-Mezeraa valley confirms the reality of a Late Glacial morphogenesis characterized by the accumulation of alluvial fans. During Holocene period, the lack of datings does not allow to recognize the stages of the palaeoclimatological models of the eastern Maghreb. Middle and Upper Holocene are well represented and allow to show the existence of a morphogenic crisis contemporary with that of the northern side of the Mediterranean during Bronze and Early Iron Ages. Evidence of a more favorable hydric balance exists about 2 400-2 200 B.P. Holocene is characterized too by three phases of downcutting in the wadis : one, poorly dated, before the Climatic Optimum, a second due to the Bronze and Early Iron Ages crisis, and the third from Medieval period up to now. Nevertheless, only two alluvial terraces were formed : the lower terrace whose very complex genesis began before 5830 B.P. and ended after 1350 B.P., and the very low terrace.

Citer ce document / Cite this document :

Ballais Jean-Louis, Benazzouz Mohamed T. Données nouvelles sur la morphogenèse et les paléo-environnements tardiglaciaires et holocènes dans la vallée de l'oued Chéria-Mezeraa (Nemencha, Algérie orientale). In: Méditerranée, Tome 80, 3-4-1994. Géographie physique de l'Algérie orientale. pp. 59-71.

doi : 10.3406/medit.1994.2859

http://www.persee.fr/web/revues/home/prescript/article/medit_0025-8296_1994_num_80_3_2859 


\section{Données nouvelles sur la morphogenèse et les paléoenvironnements tardiglaciaires et holocènes dans la vallée de l'oued Chéria-Mezeraa (Nemencha, Algérie orientale)}

\section{Jean-Louis BALLAIS* Mohamed T. BENAZZOUZ**}

\begin{abstract}
Résumé - L'étude de l'évolution de la vallée de l'oued Chéria-Mezeraa confirme l'existence d'une morphogenèse tardiglaciaire caractérisée par l'accumulation de cônes alluviaux. L'Holocène moyen et supérieur est bien représenté et permet la mise en évidence d'une crise morphogénique entre environ 4000 et 2400 B.P., et d'une période à bilan hydrique plus favorable que l'actuel vers $2400-2200$ B.P. L'Holocène se caractérise également par l'existence de trois phases d'entaille des cours d'eau mais elles $n^{\prime}$ individualisent que deux terrasses.

Abstract - The study of the evolution of the wadi Cheria-Mezeraa valley confirms the reality of a Late Glacial morphogenesis characterized by the accumulation of alluvial fans. During Holocene period, the lack of datings does not allow to recognize the stages of the palaeoclimatological models of the eastern Maghreb. Middle and Upper Holocene are well represented and allow to show the existence of a morphogenic crisis contemporary with that of the northern side of the Mediterranean during Bronze and Early Iron Ages. Evidence of a more favorable hydric balance exists about $2400-2200$ B.P. Holocene is characterized too by three phases of downcutting in the wadis: one, poorly dated, before the Climatic Optimum, a second due to the Bronze and Early Iron Ages crisis, and the third from Medieval period up to now. Nevertheless, only two alluvial terraces were formed: the lower terrace whose very complex genesis began before 5830 B.P. and ended after 1350 B.P., and the very low terrace.
\end{abstract}

Il y a près de 20 ans, une ébauche de la morphogenèse holocène dans la région de Chéria a été présentée (BALLAIs, 1976), constituant une partie des travaux d'une équipe internationale pluridisciplinaire (Lubell et al., 1975, 1976). C'était alors une étude pionnière pour le Maghreb oriental où, à l'exception de la Tunisie présaharienne (COQUE, 1962), la morphogenèse holocène était pratiquement inconnue. A la suite du développement des études sur cette période, en Afrique du Nord, mais aussi dans le Midi méditerranéen français et, d'une façon plus générale, dans l'ensemble du bassin méditerranéen, l'étude de la vallée de l'oued Chéria-Mezeraa a été reprise. S'appuyant sur un programme financé par le C.N.R.S., elle a permis de réaliser de nouvelles analyses géochimiques et palynologiques, d'obtenir de nouvelles datations isotopiques ainsi que des déterminations des industries préhistoriques et des céramiques. Il est devenu ainsi possible de replacer cette évolution dans celle du Maghreb oriental (BALLAIs, 1991b, 1992a, 1992b).

En Algérie orientale, non loin de la frontière tunisienne (fig. 1), la région de Chéria, au climat méditerranéen semi-aride, se caractérise par la juxtaposition de vastes cuvettes orientées SW-NE et délimitées par des hauteurs étroites et peu élevées $(1300 \mathrm{a} 1700 \mathrm{~m})$, caractéristiques du contact entre les Nemencha s.s., au sud, les Monts de Tébessa à l'est (KALlA-Dridi, 1994) et les Hautes Plaines constantinoises,

\footnotetext{
* UFR des Sciences Géographiques et de l'Aménagement, Université de Provence et URA 903 CNRS (France).
}

** Institut des Sciences de la Terre, Université de Constantine (Algérie). 


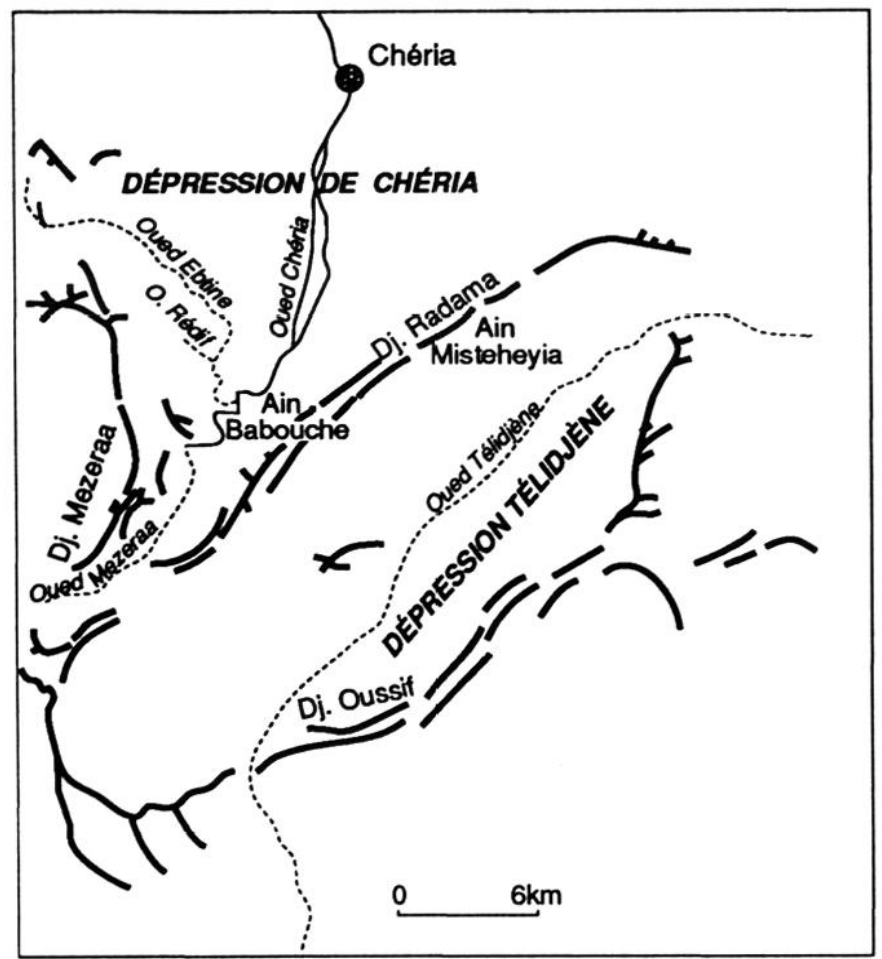

Fig. 1 - CARTE DE LOCALISATION au nord. Il s'agit, en général, de vastes combes développées dans les marnes du Crétacé supérieur aux dépens desquelles se sont élaborées les formes et formations quaternaires. Le long des cours d'eau, rivières pérennes comme l'oued ChériaMezeraa ou véritables oueds, le plus souvent, se sont conservées des formes tardiglaciaires et holocènes.

\section{1 - FORMES ET FORMATIONS TARDIGLACIAIRES ET HOLOCĖNES}

La forme majeure est constituée par une basse terrasse, très constante, souvent seule, délimitant le lit majeur des cours d'eau. Elle est accompagné, de temps en temps, d'une très basse terrasse et, très peu souvent, de cônes alluviaux latéraux construits par un affluent.

\subsection{Les cónes alluviaux antérleurs à la basse terrasse principale}

Ces cônes, très rares, ne peuvent être bien caractérisés que sur la rive droite de l'oued ChériaMezeraa dans les gorges qu'il taille, au sud d'Aïn Babouche. Le plus net est localisé à environ $200 \mathrm{~m}$ à l'amont de la coupe type de la basse terrasse (Ballais, 1976), à $1020 \mathrm{~m}$ d'altitude où un affleurement du substratum calcaire le protège de l'entaille de l'oued. A la suite de W. FARRAND et al. (1982 et in litteris), on peut décomposer les $7 \mathrm{~m}$ visibles de l'accumulation en trois membres (fig. 2).

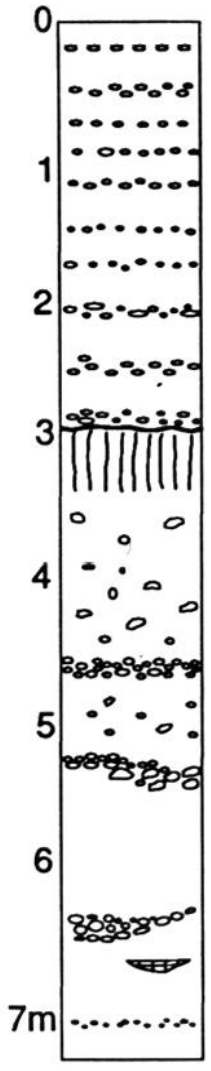

Fig. 2 - COUPE DU CÓNE TARDIGLACIAIRE D'UN AFFLUENT DE RIVE DROITE DE L'OUED CHERIA MEZERAA
1.1.1. Le membre inférieur: à la base, environ $2 \mathrm{~m}$ de sables brun jaunâtre avec des lentilles de graviers et de cailloux, calcaires comme tous les dépôts grossiers de la vallée, à l'exception des silex.

A quelques décimètres au-dessus du lit mineur actuel, s'observent des cailloux craquelés par le feu et des restes de foyers en place, riches en cendres et en charbons de bois datés au ${ }^{14} \mathrm{C} 11870 \pm 290$ B.P. (SMU 738) et $11590 \pm 100$ B.P. (SMU 655).

Une industrie sur silex, non identifiable mais pré-capsienne selon D. LuBELl (in litteris), accompagne ces foyers. En 1987, nous avons effectué deux nouveaux prélèvements de cette industrie dont les éléments ont été déterminés par Colette Roubet (Institut de Paléontologie Humaine, Paris). Celui effectué $1 \mathrm{~m}$ sous les foyers, a livré 16 pièces dont un nucleus à enlèvements centripètes, un grattoir (?) sur lame épaisse, une pièce à coche (en grès ?), une lame retouchée, une lame brute, un croissant (?) bifacial, quatre éclats bruts, un gros éclat retouché, une longue lame brute, deux éclats sur lames et un éclat retouché sur lame couvert de cacholong blanc. Le nouveau prélèvement dans les foyers est composé de deux gros éclats, deux petits éclats sur lame, un éclat retouché (?) et deux pièces irrégulières.

Deux prélèvements effectués également à $1 \mathrm{~m}$ sous les foyers pour la recherche d'éventuels pollens ont été analysés par Dominique Jouly (Laboratoire de Géologie du Quaternaire du C.N.R.S., Marseille-Luminy). Le premier, constitué de sable argileux, contenait 8 grains de pollens dont 1 Compositae, 1 Cyperaceae, 1 Gramineae, 1 Nyctaginaceae, 2 Pterydophytae monoletes et 2 indéterminables. Le second, formé par le contenu de coquilles de gastéropodes, est encore plus pauvre : 2 Amaranthaceae/Chenopodiaceae et 3 Compositae. 


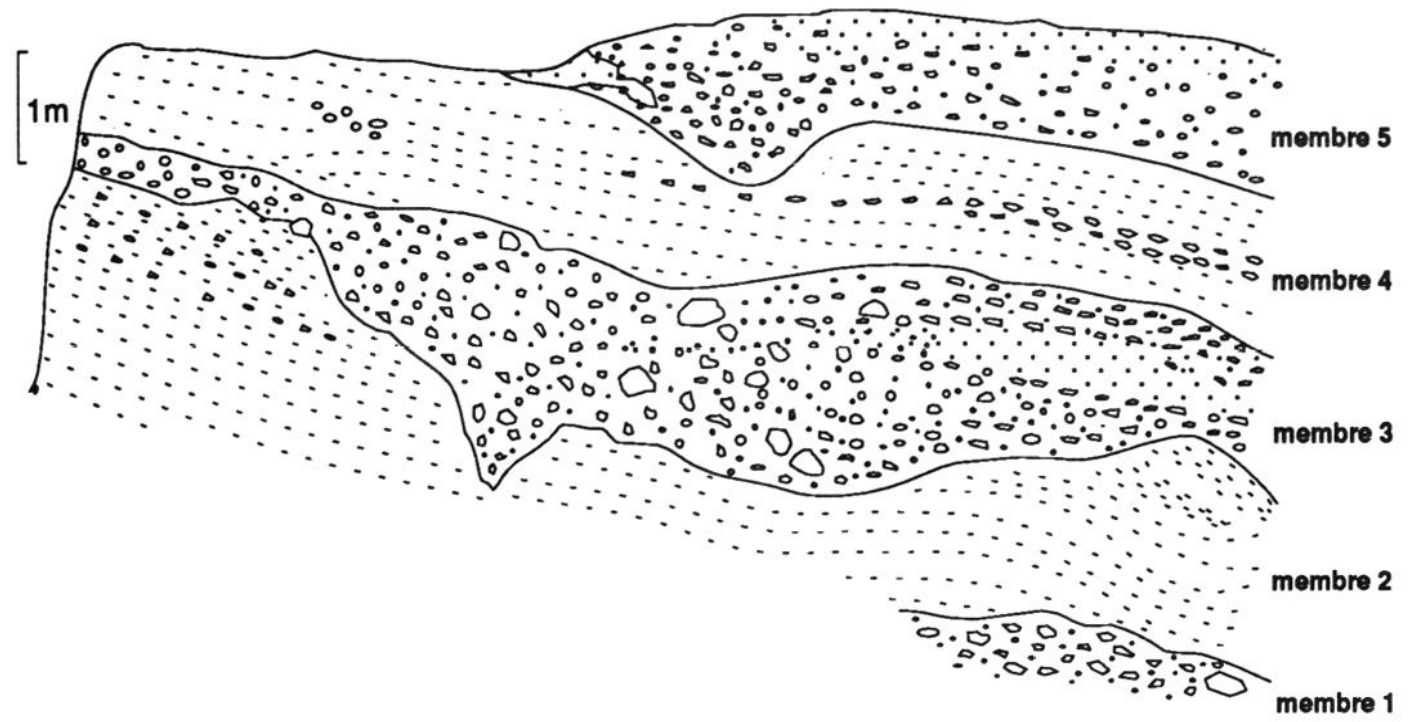

Fig. 3 - COUPE DE LA BASSE TERRASSE DE L'OUED CHÉRIA-MEZERAA

1.1.2. Le membre moyen, épais d'un peu plus de $2 \mathrm{~m}$, est tout à fait comparable au membre inférieur, mais les passées grossières sont nettement moins nombreuses : les cailloux sont dispersés dans la masse, alors que les graviers sont organisés en lentilles, vers la base. Des fragments de coquilles d'escargots sont visibles. Ce membre se termine par une formation fine, limono-argileuse, contenant de rares graviers et galets, épaisse de $50 \mathrm{~cm}$ à $1 \mathrm{~m}$, qui pourrait être un paléosol peu développé. En effet, le tiers supérieur $(15$ à $30 \mathrm{~cm})$, beige foncé, à tâches blanches, est structuré en colonnettes de $5 \mathrm{~cm}$ de diamètre alors que les deux tiers inférieurs, brun clair, ont une structuration grumeleuse et prismatique (rayon de quelques centimètres). Il contient, en abondance, des coquilles de gastéropodes qui ont fourni un âge ${ }^{14} \mathrm{C}$ de $1730 \pm 185$ B.P. (C 3378).

1.1.3. Le membre supérieur, de même épaisseur, est constitué surtout de sable et de graviers en lits horizontaux, assez bien triés.

\subsection{La basse terrasse princlpale}

En fonction de son extension et, surtout, de sa position par rapport aux reliefs, ont peut distinguer plusieurs variétés de dépôts.

\subsubsection{La coupe type de l'oued Chéria-Mezeraa:}

Elle se situe sur la rive droite de l'oued Chéria-Mezeraa, à l'extrémité méridionale de la dépression de Chéria où la vallée se réduit à $200 \mathrm{~m}$ de large environ, au pied du djebel Mezeraa. Sur $5,90 \mathrm{~m}$, elle superpose 5 membres, de bas en haut (fig. 3, photo 1 ).

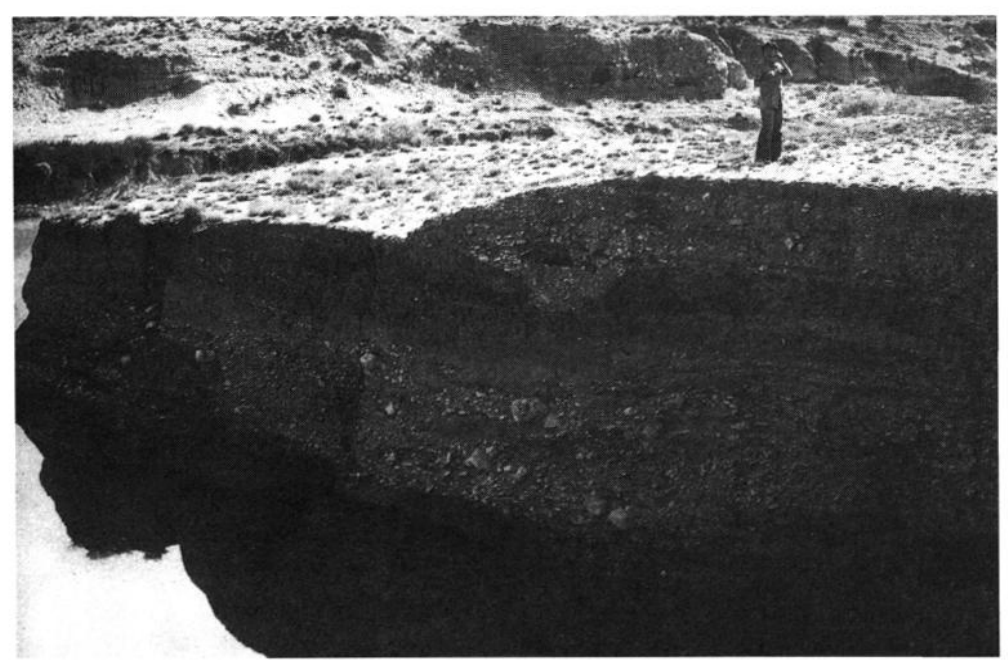

Photo 1 - COUPE-TYPE DE LA BASSE TERRASSE DE L'OUED CHÉRIA-MEZERAA.
- Le membre 1 : alternances de graviers calcaires à matrice sableuse et d'argiles beiges, visibles sur $1,18 \mathrm{~m}$; son étude n'a pu être reprise en détail au-delà de $60 \mathrm{~cm}$, car il est submergé sous la petite retenue créé par un barrage rustique. Il comprend, de bas en haut :

- graviers longs de quelques millimètres, arrondis, rarement plats, avec des cailloux peu plats et subanguleux, dans une matrice sableuse beige gris clair (5 YR 7/3); épaisseur visible : $18 \mathrm{~cm}$, - argile beige jaune (10 YR 6/4), à veines ferrugineuses, épaisseur : $22 \mathrm{~cm}$, - graviers longs de quelques millimètres, arrondis, rarement plats dans une matrice sableuse beige gris clair (5 YR 7/3) à traces ferrugineuses, épaisseur : $16 \mathrm{~cm}$, 
Tabl. 1 - SPECTRES PALYNOLOGIQUES DES DÉPÔTS DE LA BASSE TERRASSE DE L'OUED CHERIA-MEZERAA

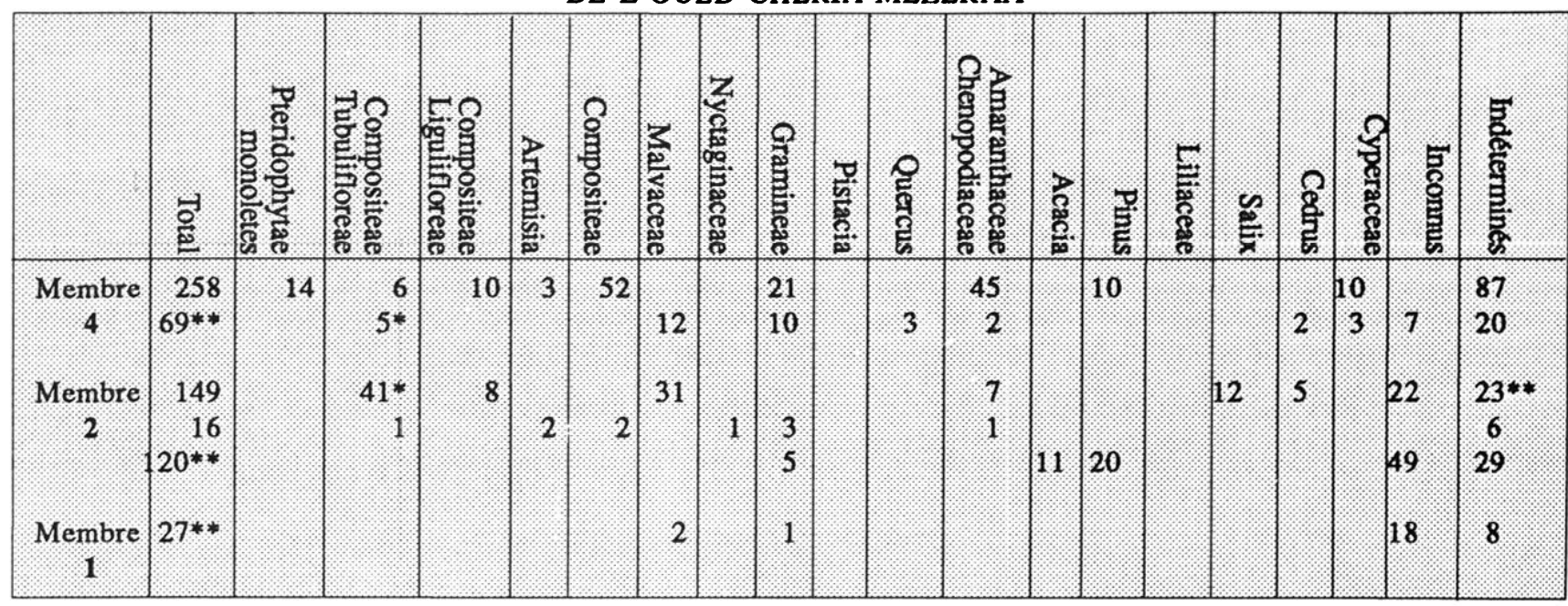

* y compris Artemisia; ** les erreurs dans les totaux proviennent de l'original (tableau III-a in LuBELl et al., 1975).

- argile beige gris foncé $(7,5$ YR 5/3) à veines ferrugineuses $(7,6<\mathrm{pH}<8)$, épaisseur : $4 \mathrm{~cm}$. Cette argile contient de rares pollens (LuBell et al., 1975): 27 grains dont $2 / 3$ inconnus et $30 \%$ indéterminés, les autres étant des pollens de Malvaceae en proportion double de ceux de Gramineae (tabl. 1), - graviers de moins de $10 \mathrm{~mm}$ de long, plats, subanguleux, avec de petits cailloux arrondis et quelques cailloux plus gros, dans une matrice limono-argileuse beige gris (7,5 YR 6/3), épaisseur : $38 \mathrm{~cm}$, - graviers denses, de moins de $10 \mathrm{~mm}$ de long, plats, subanguleux, et petits cailloux arrondis dans une matrice sablo-limono-argileuse beige gris (7,5 YR 6/3), épaisseur : $20 \mathrm{~cm}$

- Le membre 2 est formé surtout de dépôts fins, de teinte sombre, organisés en trois couches, de bas en haut :

- couche argilo-sablo-limoneuse, $(7,2<\mathrm{pH}<7,6)$ beige grisâtre $(10 \mathrm{YR} 6 / 3)$ à veines ferrugineuses orange grisâtre (5 YR 6/4) contenant un lit de graviers centimétriques et quelques galets plats $(\mathrm{Ia}=2,58)$ et subanguleux ( $\mathrm{Ie}=96$ ). Les pollens comptés ici sont plus abondants (tabl. 1$): 120$ grains mais $40,8 \%$ restent inconnus et $24,2 \%$ indéterminés. Les pollens d'arbres, avec Pinus $(16,7 \%)$ et Acacia $(9,2 \%)$, dominent les Gramineae $(4,2 \%)$ (LuBELl et al., 1975). La smectite (50\%) est le minéral argileux principal, devant la palygorskite $(25 \%)$ et la kaolinite (10\%) (tabl. 2). Epaisseur : $28 \mathrm{~cm}$,

- lentille grossière de graviers de 1 à $5 \mathrm{~mm}$ de long, plats et subanguleux avec quelques petits cailloux longs de 20 à $40 \mathrm{~mm}$, arrondis, non plats, accompagnés de quelques silex, dans une matrice sablo-limoneuse $(7,0<\mathrm{pH}<7,2)$ à veines orange grisâtre $(7,5$ YR $6 / 4)$, épaisseur : $20 \mathrm{~cm}$,

- couche de formation fine, épaisse de $76 \mathrm{~cm}$ et comprenant :

- couche limono-sableuse, grumeleuse et à fentes de dessication superficielles à la base et, vers le sommet, structuration en prismes de 5 à $10 \mathrm{~cm}$ de rayon, orange grisâtre $(5 \mathrm{YR} 6 / 3)$ à veines ferrugineuses contenant, à la base, des lentilles discontinues de graviers plats et anguleux, longs de $10 \mathrm{a} 20 \mathrm{~mm}$. Le sommet est très légèrement induré et comporte un abondant pseudo-mycélium. Un premier prélèvement pour pollens n'a fourni que 6 grains, indéterminables (LuBell et al., 1975). Un second a fourni 16 grains dont 6 indéterminables et aucun pollen d'arbres ( tabl. 1). Epaisseur : $70 \mathrm{~cm}$,

- couche discontinue d'argile sableuse $(\mathrm{pH}=7,6)$ orange grisâtre $(5 \mathrm{YR} 6 / 3)$ à veines ferrugineuses orange brun (2,5 YR 5/8), très développées quand les graviers deviennent abondants, et à tâches noires d'oxyde de manganèse. Le prélèvement pour pollens a fourni 149 grains dont $58,1 \%$ indéterminés et $14,4 \%$ inconnus. Les arbres régressent (Salix : 8,1\%, Cedrus $3,4 \%)$ et les herbacées dominent $(27,5 \%$ de Compositae tubiflora et $21 \%$ de Malvaceae) (tabl. 1). Epaisseur maximum : $6 \mathrm{~cm}$,

Ce membre a été daté de $5830 \pm 95$ B.P. (I - 7693) sur des coquilles d'une petite espèce de gastéropodes non déterminée et de $4685 \pm 95$ B.P. (Isotopes Inc., référence incomplète) environ $50 \mathrm{~m}$ plus à l'aval.

Treize échantillons de matrice ont été prélevés, tous les 10 centimètres environ, pour une étude systématique des variations des éléments (tabl. 3) qui, sur une période aussi courte que l'Holocène, pouvaient permettre de déceler, outre les variations des sources des dépôts, des évolutions géochimiques ou pédologiques 
in situ. Le second critère de choix a été la possibilité de comparaison avec les analyses déjà effectuées dans des sites proches (LuBeLl et al, 1975, 1976). Compte tenu de l'environnement très carbonaté, ce membre 2 l'est assez peu $(22,1 \%$ à $36,9 \%)$. Les taux de $\mathrm{Fe}_{2} \mathrm{O}_{3}(2,46 \%$ à $4,05 \%)$ et de $\mathrm{P}_{2} \mathrm{O}_{5}(0,58 \%$ à $1,04 \%)$ sont notables, ainsi que ceux de carbone $(0,81 \%$ à $1,07 \%)$.

Tabl. 2 - MINÉRAUX ARGILEUX DES DÉPÓTS DE LA BASSE TERRASSE DE L'OUED CHÉRIA-MEZERAA

\begin{tabular}{|c|c|c|c|c|c|c|c|}
\hline Echantillon & Kaolinite & Smectite & Illite & $\mathrm{Sm} / \mathrm{mil}$ & Chlorite & Interst. $14_{\mathrm{c}}-14_{\mathrm{v}}$ & Palygorskite \\
\hline membre 2 & 10 & 50 & 5 & $(10)$ & 5 & 5 & 25 \\
\hline membre 3 & 15 & 50 & 5. & $\left(10^{*}\right)$ & $5^{t}$ & & 25 \\
\hline membre 4 & 10 & 60 & 5 & (12) & 5 & & 20 \\
\hline membre 5 & 15 & so & $<5$ & (16) & 10 & & 25 \\
\hline
\end{tabular}

- Le membre 3 est un ensemble grossier, comblant parfois un chenal entaille dans le membre 2, presque jusqu'à sa base. Epais de $138 \mathrm{~cm}$, il est formé, de bas en haut, par 3 couches :

- nombreux cailloux longs de $20 \mathrm{~mm}$, plats et subarrondis, accompagnés de quelques cailloux de $80 \mathrm{~mm}$ de long, arrondis, non lités, et de silex, dans une matrice limoneuse abondante beige orange grisâtre (7,5 YR 6/4) à structure prismatique ; quelques Helicella. Dans la partie sommitale, apparaissent quelques galets d'argile. Le cortège de minéraux argileux est très comparable à celui du membre 2 (tabl. 2). Cependant, les interstratifiés $14 \mathrm{c}^{-14} \mathrm{v}$ disparaissent. Epaisseur : $40 \mathrm{~cm}$,

- lentille de cailloux longs de $100 \mathrm{~mm}$ plats et non plats, arrondis, avec un bloc long de $300 \mathrm{~mm}$ et des silex, mal lités, dans une matrice limoneuse peu abondante beige orange grisâtre (7,5 YR 6/4); quelques Helicella, épaisseur : $58 \mathrm{~cm}$,

- lentille de graviers et de cailloux plats (Ia $=2,52$ ), subanguleux (Ie $=96$ ), longs de $3 \mathrm{~mm}$ a $50 \mathrm{~mm}$, lités, avec quelques silex, des coquilles d'Helicella et de petits gastéropodes coniques; dans la partie centrale, une lentille d'argile limoneuse beige grisâtre (10 YR 6/3), épaisseur : $40 \mathrm{~cm}$.

Quatre échantillons, également répartis de la base au sommet, ont été également analysés (fig. 5). Alors que la médiane de la matrice du membre 2 varie entre $6 \mu \mathrm{m}$ et $22,5 \mu \mathrm{m}$, celle du membre 3 est moins fine et peut atteindre jusqu'à $302 \mu \mathrm{m}$ dans les lentilles les plus grossières. Les analyses chimiques montrent un taux de carbonates nettement plus important (de $49,4 \%$ a $65,1 \%$ ), une baisse des proportions de $\mathrm{Fe}_{2} \mathrm{O}_{3}, \mathrm{Na}^{+}, \mathrm{Cl}^{-}, \mathrm{C}$ et $\mathrm{N}$, alors que $\mathrm{P}_{2} \mathrm{O}_{5}$ se maintient.

- Le membre 4, épais de 1,10 m, est un ensemble constitué de dépôts fins, un seul membre subdivisé en trois :

- limons sableux ( $\mathrm{pH}=7,2)$ brun gris $(7,5 \mathrm{YR} 5 / 3)$, à pseudo-mycelium et structuration grumeleuse, contenant des graviers et des petits cailloux longs de 20 à $30 \mathrm{~mm}$, non plats, subanguleux et des coquilles de gastéropodes (nombreux Helicella, deux Rumina decollata). Un premier prélèvement a fourni 69 grains de pollens ( $29 \%$ indéterminés et $10,1 \%$ inconnus) : deux arbres sont représentés (Quercus : 4,3\% et Cedrus : $2,9 \%$ ), les Malvaceae redeviennent dominantes (17,5\%) devant les Gramineae (15\%) (LUBELL et al., 1975) (tabl. 1).

Un second prélèvement, plus riche (171 grains), n'a révêlé la présence que d'un seul arbre (Pinus : $5,8 \%$, mais sans exclure une possible pollution), alors que les Compositaea $(30,4 \%)$ ne précèdent que de peu les Amaranthaceae-Chenopodiaceae (26,3\%), loin devant les Gramineae (12,3\%) (tabl. 1).

La composition du cortège de minéraux argileux reste globalement stable (tabl. 2). Vers le second tiers, un tesson de céramique tournée commune grise a été trouvé. Epaisseur : $32 \mathrm{~cm}$,

- lentille de cailloux longs de $30 \mathrm{~mm}$ au maximum, plats, subanguleux, lités, dans une matrice limoneuse $(\mathrm{pH}=7)$ beige gris foncé (10 YR 4/3). Le spectre pollinique (59 grains) s'enrichit d'arbres (Pistacia, Acacia et Pinus dont le total dépasse, pour la première fois, $20 \%$ ) (tabl. 1). Epaisseur : $8 \mathrm{~cm}$,

- limons argileux $(\mathrm{pH}=7,2)$ à assez nombreuses coquilles d'Helicella beige-gris clair $(10 \mathrm{YR}$ 6/2) à la base, beige grisâtre (10 YR 6/4) au milieu et brun-gris clair (10 YR 6/2) au sommet; localement, une lentille de graviers et petits cailloux plats $(\mathrm{Ia}=2)$ et anguleux $(\mathrm{Ie}=85)$ s'intercale entre le milieu et le sommet.

Le spectre pollinique (43 grains) voit la disparition des Gramineae et le flechissement des arbres bien que les pourcentages cumulés de Quercus, d'Acacia et de Cedrus dépassent encore $16 \%$ (tabl. 1 ). Un second tesson de céramique commune grise, probablement tournée, a été trouvé dans cette couche. Epaisseur : $70 \mathrm{~cm}$. 


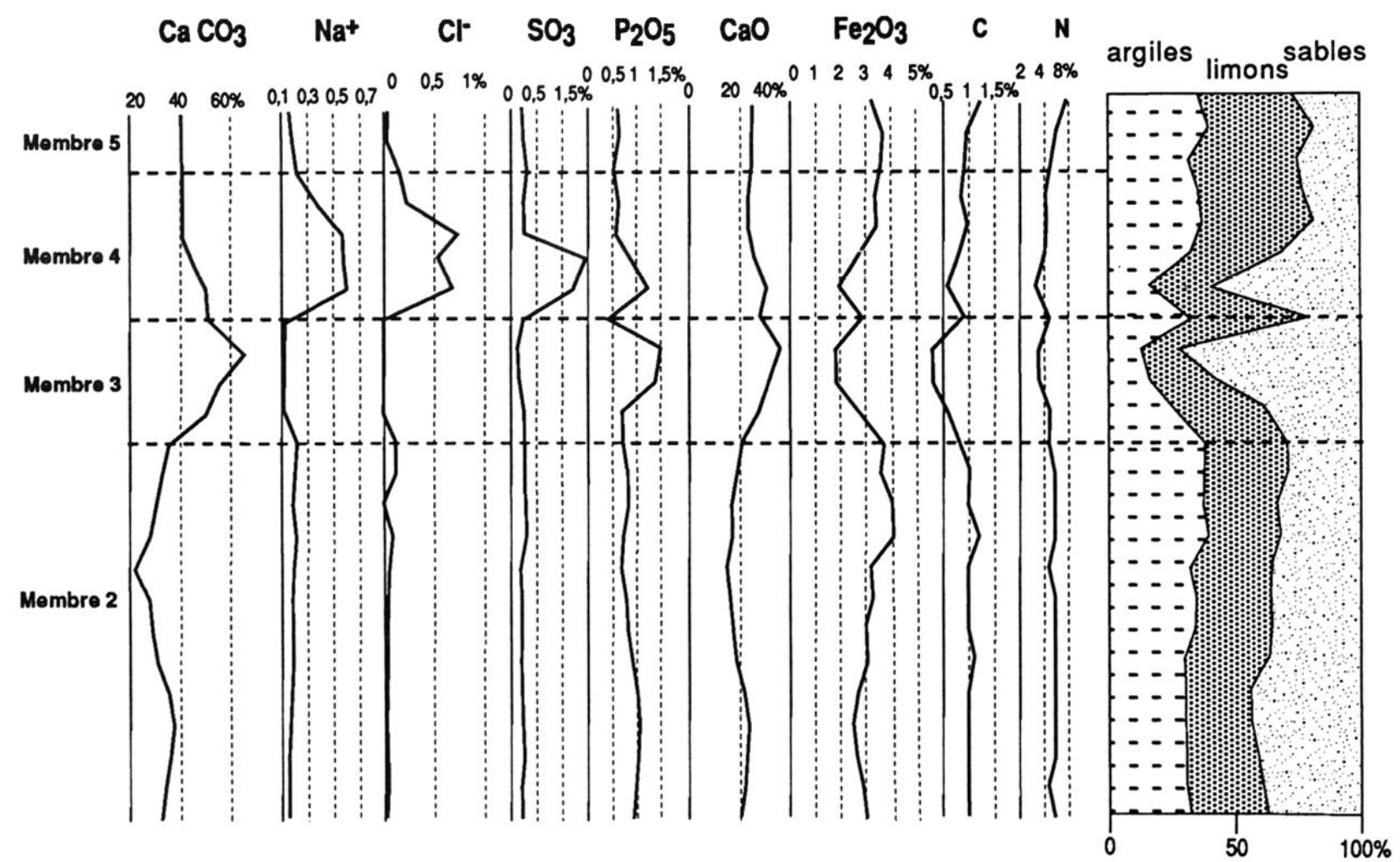

Fig. 4 - CARACTÉRISTIQUES GÉCHIMIQUES ET GRANULOMÉTRIQUES DES DÉPÔTS DE LA BASSE TERRASSE DE L'OUED CHERIA-MEZERAA

Quatre échantillons, régulièrement espacés de la base au sommet, ont été analysés (fig. 5). Leur médiane varie de $5,3 \mu \mathrm{m}$ à $71 \mu \mathrm{m}$. Leurs caractéristiques géochimiques sont intermédiaires entre celles du membre 2 et celles du membre 3 avec, cependant, le maximum de $\mathrm{Na}^{+}$, de $\mathrm{Cl}^{-}$et de $\mathrm{SO}_{3}$.

Deux datations au ${ }^{14} \mathrm{C}$ ont été obtenues, sur coquilles de gastéropodes, vers la base du membre : $2260 \pm 30$ B.P. (SMU 1119) sur un mélange de coquilles d'Helix melanostoma et d'Otala sp., et $2270 \pm 80$ B.P. (1 - 9833).

- Le membre 5 constitue le sommet de l'accumulation. Il n'est représenté que localement et repose en discordance sur le membre 4, remplissant, en particulier, une dépression de forme très régulière interprétée comme un canal d'irrigation. Epais de $90 \mathrm{~cm}$, il comprend deux couches :

- Ientille limoneuse brun gris clair (10 YR 5/3) contenant une grosse lentille de graviers longs de 5 à $10 \mathrm{~mm}$ plats et subanguleux, des cailloux plats longs de 50 à $70 \mathrm{~mm}$ et de gros cailloux non plats ou des blocs qui atteignent jusqu'à $400 \mathrm{~mm}$ de long; nombreuses coquilles de gastéropodes (Helicella très dominants). Les proportions des minéraux argileux varient peu (tabl. 2). Epaisseur : $46 \mathrm{~cm}$,

- limons beige grisâtre (10 YR 6/3), à coquilles d'Helicella et racines carbonisées, épaisseur : $44 \mathrm{~cm}$. Un échantillon prélevé dans chacune des deux couches a été analysé (fig. 5). Leur médiane reste très faible $(3,6 \mu \mathrm{m}$ à $5,2 \mu \mathrm{m})$ et ils se caractérisent par le maximum de $\mathrm{C}$ et de $\mathrm{N}$, le minimum de $\mathrm{Cl}^{-}$et de $\mathrm{P}_{2} \mathrm{O}_{5}$, ainsi que par des taux de $\mathrm{CaCO}_{3}$ et de $\mathrm{Fe}_{2} \mathrm{O}_{3}$ proches de ceux du membre 2 .

Ce membre 5 a également été daté au ${ }^{14} \mathrm{C}$, sur charbons de bois : $1450 \pm 90$ B.P. (SMU 1441) et $1350 \pm 70$ B.P. (Beta-2734) pour le même échantillon et $1730 \pm 185$ B-P. (C 3378) sur coquilles de gastéropodes. Ce membre terminal est couvert d'un reg de cailloux calcaires.

Le long de l'oued Chéria-Mezeraa, vers l'amont, l'altitude relative de la basse terrasse principale diminue et, vers Aïn Babouche (fig. 1), elle se confond avec le lit majeur. Aïn Babouche marque donc la limite amont actuelle de l'érosion régressive exercée par la rivière.

\subsubsection{Les autres coupes :}

Un premier type comprend une succession de faciès très comparable à celle de la coupe type, mais compliquée par le remaniement, dans le membre 2, d'escargotières capsiennes proches, par exemple à Aïn Misteheyia ou dans l'oued Redif (fig. 1 et 4) (Lubell et al., 1975, 1976). 


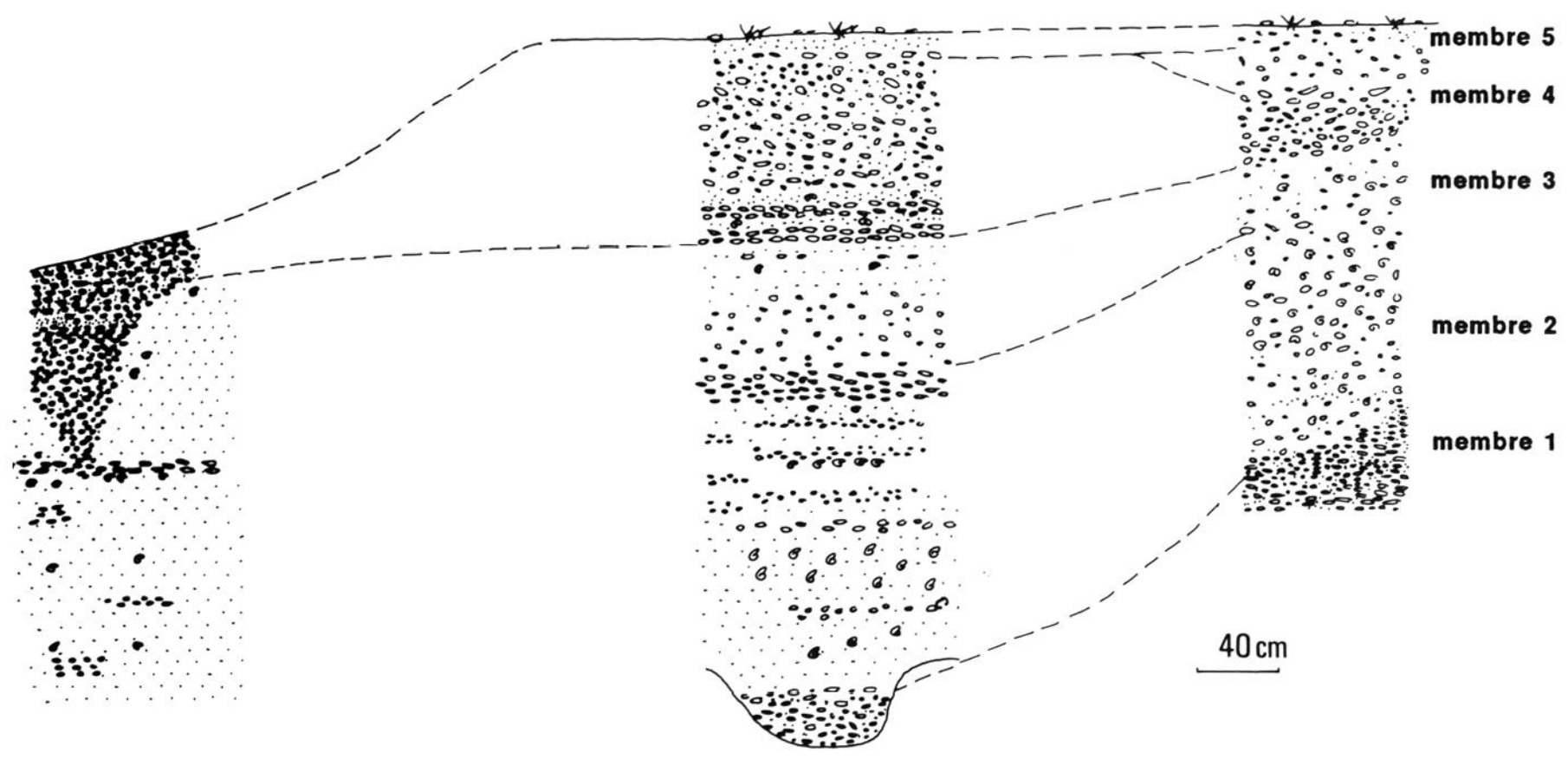

Fig. 5 - COUPE DE LA BASSE TERRASSE DE L'OUED REDIF

Un second type correspond au fond des dépressions où les dépôts sont plus fins et moins contrastés comme dans l'oued Ebtine (fig. 1).

Au total, on peut distinguer, en général :

- à la base, un membre grossier ou mixte, non daté, discordant sur le substratum ou sur les formations du Pléistocène supérieur,

- un membre constitué du faciès fin majeur gris brun, souvent riche en coquilles de gastéropodes et remaniant le matériel d'escargotières capsiennes, matériel daté de $7690 \pm 120$ B.P. (1 - 7692) et de $7340 \pm 115$ B.P. (1 - 7694) dans l'oued Redif (fig. 4). Outre les datations de la coupe type, il a fourni une date ${ }^{14} \mathrm{C}$ de $6950 \pm 60$ B.P. (SMU 688) dans l'oued Oussif, au sud de la dépression de Télidjène (fig. 1),

- un membre grossier supérieur, non daté, très nettement discordant sur le membre précédent qu'il ravine, - un membre supérieur de faciès fin ou mixte, peu épais, discordant lui aussi et daté au ${ }^{14} \mathrm{C}$, sur charbons de bois, $2590 \pm 90$ B.P. dans l'oued Regada au centre de la combe de Télidjène (FARrand et al., 1982),

- enfin, un membre terminal, de faciès varié.

\subsection{Les formes et formations assoclées :}

\subsubsection{Les cones alluviaux :}

Ils ne sont notables qu'au pied des principaux reliefs. Les plus nets se situent sur les affluents de l'oued Chéria-Mezeraa. C'est ainsi que, au niveau de la coupe type, mais sur la rive gauche, les dépôts grossiers d'un cône alluvial ravinent le membre 2 et viennent s'interstratifier dans le membre 3.

\subsubsection{Les éboulis :}

Pendant l'accumulation de la basse terrasse principale, l'évolution des versants semble avoir été minime : la plupart des versants réglés du Pléistocène moyen ont été peu retouchés. Cependant, des corniches ont recommencé à fonctionner, particulièrement à proximité de l'oued Chéria-Mezeraa. En effet, la coupe type montre, tout à fait à la base, de gros blocs de calcaire anguleux, basculés vers le lit de la rivière, et issus de la corniche située immédiatement au-dessus. A l'amont, sur la rive gauche, une grande corniche calcaire, orientée au nord, domine la bassc terrasse; le passage de l'une à l'autre se fait par un cône d'éboulis comprenant d'énormes blocs de plusieurs mètres cubes, posés à plat, qui passent dans la terrasse dont ils émergent encore en partie à l'amont. Ils ont écrasé un site capsien. 
Donc, le membre 1, mais surtout le membre 3, ont été formés, non seulement par des apports longitudinaux importants, mais aussi par des apports transversaux non négligeables.

\subsubsection{Les encroutements :}

Au contraire du Pléistocène moyen, ils sont toujours exceptionnels et limités; tout au plus, dans les bas-fonds, des nodules de calcaire se sont parfois individualisés. Par contre, lorsqu'une escargotière capsienne repose partiellement sur la roche calcaire en place, comme au djebel Misteheyia (piémont du djebel Radama, fig. 1), des enduits calcaires ont cimenté des coquilles de gastéropodes ou des silex taillés demeurés dans les lapiès.

\subsection{Les très basses terrasses:}

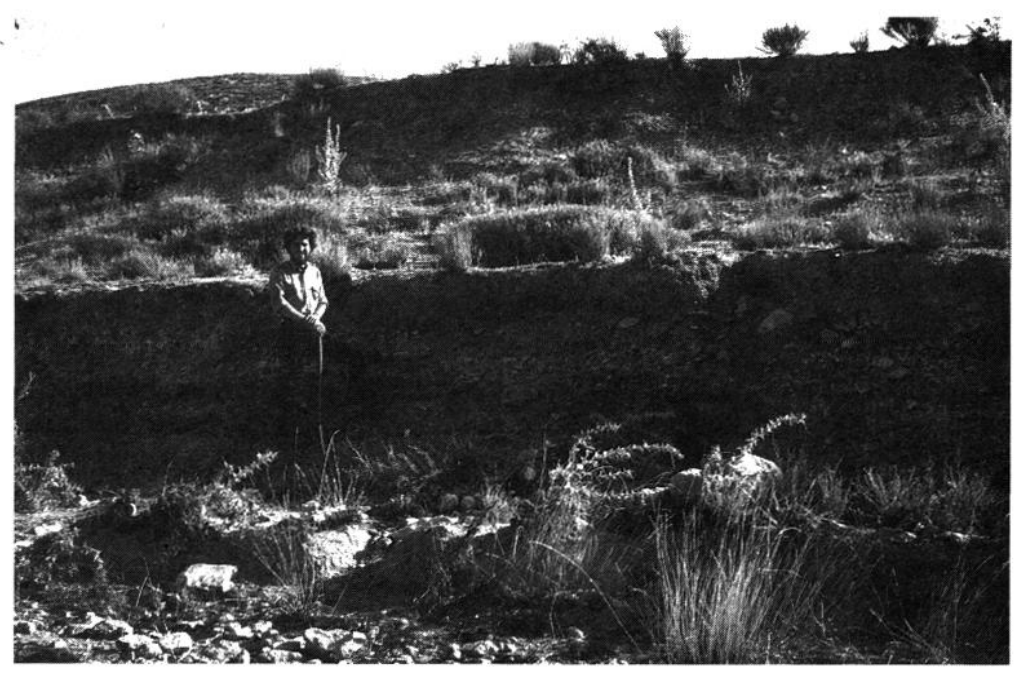

Photo 2 - LA BANQUETTE SUPÉRIEURE OU TRES BASSE TERRASSE DE L'OUED CHERIA-MEZERAA.

A l'arrière-plan, la basse terrasse.

Leur extension est extrêmement réduite. Sur les principaux oueds, elles apparaissent nettement, à la faveur d'une entaille régressive récente. C'est particulièrement le cas sur l'oued Chéria-Mezeraa, à l'aval de la coupe type. Là, s'individualisent progressivement vers l'aval deux banquettes. La banquette supérieure, d'abord à fleur d'eau à l'aval, atteint rapidement 2 à $3 \mathrm{~m}$ d'altitude relative et reste emboîtée de 3 à $5 \mathrm{~m}$ dans les dépôts de la basse terrasse (photo 2). Elle comporte une couverture alluviale, épaisse d'au moins $50 \mathrm{~cm}$, formée, à la base, de galets calcaires plats $(\mathrm{Ia}=2,04)$ et subanguleux $(\mathrm{Ie}=107)$, parfois tachés de rouille et d'oxyde de manganèse, à matrice peu développée, recouverts par une formation limoneuse brune à très rares cailloux. On peut penser qu'il s'agit d'une terrasse vraie, ancien lit majeur exondé définitivement depuis peu.

La banquette inférieure, discontinue, haute de seulement $50 \mathrm{~cm}$, ne comporte que des galets sans matrice. Parfois fonctionnelle lors des crues, elle occupe une position intermédiaire entre un lit majeur et une terrasse.

\section{2 - MORPHOGENĖSE ET PALÉOENVIRONNEMENTS}

L'accumulation de cônes au Pléistocène supérieur terminal confirmerait l'existence d'un véritable Tardiglaciaire au Maghreb oriental (Ballais, 1992a) alors que l'évolution de la morphogenèse et des paléoenvironnements au cours de l'Holocène s'accorde bien avec celle de la Tunisie (BALLAIS, 1991b, 1992b, 1993) avec quelques nuances.

\subsection{L'accumulation des cónes tardiglaciaires :}

Ces cônes se logent dans une entaille vigoureuse qui a éliminé, au moins dans les gorges, les témoins d'un glacis du Pléistocène supérieur bien visible dans les dépressions (Lubell et al., 1975). L'accumulation reprend donc pendant l'Allerơd, pulsation humide, ou peut-être même dès le Bôlling puisque au moins $1 \mathrm{~m}$ de dépôts seraient antérieurs à $11897 \pm 286$ B.P. Les pollens déterminés sont en trop petit nombre pour permettre une conclusion argumentée.

Mais des incertitudes persistent car aucune discordance n'apparaît entre le membre inférieur et le membre moyen qui se termine par le paléosol probable daté $1730 \pm 185$ B.P. A moins que, malgré les affirmations de certains préhistoriens, les foyers à industrie pré-capsienne ne soient pas en place et soient remaniés dans les alluvions, comme cela s'est produit également, et au moins à deux reprises, pour les gisements capsiens (LuBELL et al., 1975). L'incertitude demeure également pour situer la place éventuelle du Dryas récent, encore mal connu et dont les caractéristiques, au moins après 10600 B.P., sont celles d'une phase plutôt humide (Ballais, 1992a). 


\subsection{Les Incertitudes de l'Holocène Inférleur}

L'optimum hydrologique du début de l'Holocène, bien marqué en Tunisie saharienne (PetrT-MaIRE et al., 1991), semble avoir êté très discret dans la région de Chéria où il ne se marque, dans l'état actuel de nos connaissances, que par des dépôts marécageux à gley datés, sur gastéropodes (surtout Helicella setifensis), de $9230 \pm 80$ B.P. (SMU 711), en bordure de l'oued Télidjène.

Face aux incertitudes chronologiques, il n'est pas possible de discuter, de façon précise, des conditions qui permettent, ensuite ou avant, $l^{\prime}$ entaille dans laquelle s'accumulera la basse terrasse principale et, en particulier, le rôle d'une phase sèche bien reconnue maintenant en Tunisie présaharienne par exemple, entre 7500 B.P. et 7000 B.P. (Ballais, 1991b) et qui pourrait être un peu plus précoce, autour de 8125 \pm 125 B.P., dans la combe de Télidjène (LuBell et al., 1982-83).

\subsection{Le dépót de la basse terrasse principale}

Comme dans le cas des cônes, la formation de la terrasse correspond à une fourniture de matériel détritique suffisante pour saturer les écoulements et provoquer une accumulation généralisée. Cependant, dans le détail, les nuances sont considérables.

\subsubsection{Le dépôt du membre inférieur grossier :}

Les graviers et petits cailloux qui le constituent avaient été attribués à une reprise de la désagrégation mécanique et, probablement, de la gélifraction (Ballars, 1976). En fait, ils peuvent aussi dériver de la couverture du glacis du Pléistocène supérieur et, ici aussi, le trop petit nombre de pollens déterminés ne permet pas d'apporter d'indications indirectes concernant le climat. Enfin, l'incertitude chronologique est trop grande pour que la comparaison avec l'Holocène inférieur et moyen de Tunisie soit possible.

Malgré ces incertitudes, il est très vraisemblable que le dépôt d'au moins la partie supérieure de ce membre inférieur soit contemporain des Capsiens qui fréquentèrent la région d'environ 9500 B.P. a 6500 B.P. (Lubeli et al., 1975). Dans le bassin méditerranéen occidental, il est généralement admis que les populations épipaléolithiques, pré-néolithiques, vivant de chasse, de pêche et de cueillette, n'ont pas eu suffisamment d'impact sur les géosystèmes pour provoquer une morphogenèse accélérée. Or, la période capsienne se caractérise par une extraordinaire densité des sites (Grebenart, 1975). Cependant, ces sites, très probablement, n'ont été occupés que par intermittence, et par de petits groupes (Lubell et al., 1975). Enfin, le faciès de ce membre inférieur n'est pas caractéristique de celui de dépôts issus de l'érosion de sols.

Au total, les caractéristiques granulométriques et sédimentologiques indiquent une compétence modérée des écoulements et, probablement, un régime hydrologique assez régulier.

\subsubsection{Le dépót du membre fin majeur :}

Ses sables limoneux, ses argiles gris brun, ses graviers et galets rares indiquent des écoulements à plus faible compétence et un régime plus régulier que précédemment. En particulier, la généralisation de ce dépôt, y compris dans les terrasses de cours d'eau qui sont, actuellement, de véritables oueds, indique l'extension d'un régime pérenne à de nombreux cours d'eau intermittents.

Deux spectres polliniques de la coupe type sont utilisables. Celui de la couche inférieure (tabl. 1) se caractérise par la forte représentation des arbres, Pinus et Acacia, qui regroupent plus de $25 \%$ des pollens et qui pourraient indiquer une forêt claire peu différente de la forêt «climacique» actuelle, d'autant plus que des charbons de Quercus ilex et d'un conifere (Cupressus?) les accompagne (Ballais, 1976). Cependant, la présence d'Acacia, aujourd'hui représenté plus au sud, pourrait indiquer une nuance climatique plus chaude qu'aujourd'hui. Celui de la couche supérieure (tabl. 1) montre une régression des arbres. Dans ce contexte, l'apparition de Cedrus, qui aime la neige et n'est plus représenté actuellement que dans le massif des Aurès, à $100 \mathrm{~km}$ plus à l'ouest (BallaIs, 1984), pourrait marquer un refroidissement. L'apparition de Salix, caractéristique du lit majeur, et la prédominance des herbacées indiquerait le début d'un assèchement. Les spectres polliniques de l'oued Redif (Lubell et al., 1976) regroupent de 146 à 244 pollens, mais ils ne sont pas bien datés. Cependant, ils doivent être globalement contemporains du membre 2. Ils montrent, de la base au sommet, une tendance à l'augmentation du pourcentage d'arbres (de 6,2 à 15,7\%) mais sans atteindre les taux de l'oued Chéria-Mezeraa. Peut-être faut-il voir dans ces nuances, le rôle des facteurs édaphiques locaux sur une ripisylve, toujours mieux fournie le long de l'oued Chéria-Mezeraa pérenne? Les Helicella abondantes dans ce membre vivent encore aujourd'hui sur les joncs, au bord des rivières. 
Les dépôts accumulés dans ce membre proviennent du remaniement d'escargotières capsiennes proches mais aussi, et surtout, de sols développés sous forêt méditerranéenne claire. Cette origine expliquerait la présence d'interstratifiés $14 c^{-14} v$ qui disparaissent ensuite, le plus faible rapport smectite/illite $(=10)$, les plus faibles taux de carbonate de calcium de tout l'Holocène, le maximum de $\mathrm{Fe}_{2} \mathrm{O} 3$, et des pourcentages importants de $\mathrm{P}_{2} \mathrm{O}_{5}$ et $\mathrm{C}$.

Le climat était donc plus humide que l'actuel ou, plus précisément, le bilan hydrique était plus favorable, par suite d'une meilleure répartition des précipitations dans l'année. Dans le détail, après un optimum thermique, les températures ont pu baisser pendant que la sécheresse progressait.

Au total, la période de dépôt du membre 2 offre donc toutes les caractéristiques de l'optimum climatique holocène. Son âge, contemporain de la fin de l'Atlantique européen, a pu paraître tardif (LuBELL et al., 1975) mais il se corrèle parfaitement avec les conclusions tirées de l'étude des pollens du marais d'Oum el-Khaled, alimenté par l'oued Chéria-Mezeraa, environ $6 \mathrm{~km}$ à l'aval de la coupe type (RrTchr, 1984) qui montrent l'existence d'une forêt de Pinus et Quercus, sous un climat plus humide que l'actuel (au moins 400 à $500 \mathrm{~mm} /$ an contre $350 \mathrm{~mm} /$ an aujourd'hui), depuis au moins $5050 \pm 100$ B.P. et jusqu'à environ $4100 \pm 80$ B.P. (RrTche, 1984). Cette chronologie confirme la zonation établie dans l'ensemble du Maghreb oriental mais laisse pendant le problème de l'absence, au moins apparente, de l'optimum hydrologique du début de l'Holocène (Ballais, 1992b), perceptible cependant dans certains sites capsiens comme celui d'Aïn Misteheyia (LuBell et al., 1975).

A la différence des sites capsiens, les sites néolithiques, contemporains de ce membre 2 , sont rares dans l'Atlas saharien algérien oriental où ils sont tous localisés sous grotte (RouBet, 1979). Même en admettant que les Néolithiques les plus anciens aient cohabité avec les Capsiens tardifs, rien n'indique une pression anthropique accrue sur les géosystèmes.

\subsubsection{L'entaille intra-terrasse et le membre grossier majeur :}

L'entaille très profonde pratiquée dans le membre 2 a été observée également dans la basse terrasse de l'oued Redif (fig. 5), mais aussi dans la basse terrasse de plusieurs oueds des Aurès (Ballais, 1984). Il ne s'agit donc pas seulement d'un épiphénomène local. Après la phase d'écoulements lents dans un lit mineur large et peu profond, elle indique la réapparition d'un écoulement très étroitement chenalisé dans un lit mineur étroit et profond.

Puis ce chenal est comblé par des dépôts grossiers qui débordent ensuite, indiquant une nouvelle phase d'extension latérale du lit mineur. L'accumulation moyenne annuelle est de l'ordre de $0,4 \mathrm{~mm}$, soit une vitesse faible (Ballais, 1991c). La masse de certains blocs déposés et le litage plus fruste indiquent des débits maxima beaucoup plus puissants que précédemment ainsi que des écoulements plus spasmodiques.

Les caractéristiques géochimiques montrent une évolution moins importante que dans le membre précédent avec le maximum de $\mathrm{CaCO}_{3}$ et les minima de $\mathrm{Fe}_{2} \mathrm{O}_{3}, \mathrm{Na}^{+}, \mathrm{Cl}^{-}$et $\mathrm{C}$. Ce ne sont donc pas seulement les sols qui sont alors décapés.

Cette phase de morphogenèse fluviatile vigoureuse n'est pas directement datée. Elle se place entre la fin de l'optimum holocène, ici vers 4000 B.P. au plus tôt, et avant 2300 B.P.. Elle est donc contemporaine du Subboréal européen et pourrait correspondre à la crise de l'âge du Bronze et du Fer mise en évidence, en particulier, dans le Midi méditerranéen français (JORDA et al., 1993 ; Ballais et al., 1993). Le bilan hydrique aurait été moins favorable et saisonnièrement plus contrasté qu'auparavant. Le climat, en particulier, aurait vu réapparaître des pluies intenses, favorisant des crues violentes à forte compétence. La désagrégation mécanique a pu reprendre sur certains versants mal végétalisés, en liaison avec un rafrâichissement hivernal du climat, multipliant les cycles gel-dégel.

\subsubsection{Le membre supérieur fin :}

Les caractéristiques générales de ce membre se rapprochent de celles du membre 2. En particulier, la finesse des dépôts, bien organisés en lits continus, évoque des écoulements lents, dans un lit large et peu encaissé. La vitesse d'accumulation moyenne annuelle est comprise entre $1,4 \mathrm{~mm}$ et $2,2 \mathrm{~mm}$, soit comparable à celle de la basse terrasse holocène préhistorique au Maghreb oriental (Ballais, 1991c). Les dépôts présentent, certes, le maximum de $\mathrm{Na}^{+}$, de $\mathrm{Cl}^{-}$et de $\mathrm{SO}_{3}$, mais aussi le minimum d'azote et des taux de $\mathrm{CaCO}_{3}$ élevés. Dans le détail, $\mathrm{Fe}_{2} \mathrm{O}_{3}, \mathrm{CaCO}_{3}$ et $\mathrm{C}$ se concentrent dans le haut du dépôt, au contraire de tous les autres corps, à l'exception de l'azote dont les variations ne sont pas significatives (fig. 4). On peut interpréter cette répartition, combinée aux variations de couleurs, comme le résultat d'un début de pédogenèse. Un autre sol, au sommet de la terrasse de l'oued Regada (fig. 1) a été daté de $2590 \pm$ 90 B.P. (FARRAND, in litteris). 
Les pollens sont encore peu abondants, mais nettement plus que dans les membres inférieurs et permettent de mettre en évidence, comme dans le membre 2, la possibilité de l'existence d'une forêt claire à Pistacia, Acacia et Pinus, Cedrus poussant sur les plus hauts sommets, contemporaine des couches moyennes et supérieures. Cependant, la proportion de pollens d'arbres n'atteint pas celle représentée dans le membre 2. Par ailleurs, la couche basale présente un spectre caractéristique d'une steppe pauvre en Armoise selon les critères d'Annick BRUN $(1985)(A / D=0,06)$ dans lequel le seul arbre représenté est Pinus, dont on connaît la capacité des pollens à se déplacer facilement et loin et qui, de plus, pourrait être le résultat d'une pollution.

Somme toute, les conditions hydrologiques et climatiques se rapprochaient de celles qui régnaient pendant l'accumulation du membre 2, mais dégradées dans le sens de la sécheresse. On peut envisager, par exemple, une augmentation de l'évaporation estivale, mais les hivers devaient rester assez frais pour permettre le maintien de Cedrus en altitude.

Ce membre est donc contemporain d'une phase plus humide maintenant nettement caractérisée dans le Maghreb oriental vers 2400 - 2200 B.P. (Ballais, 1992c) et qui marque le début du Subatlantique.

\subsubsection{Le membre 5 :}

Ses caractéristiques sédimentologiques sont très proches de celles du membre 4 et indiquent également des écoulements à faible compétence et un régime peu contrasté.

Ses caractéristiques géochimiques sont plus ambiguès car elles regroupent, à la fois, des éléments qui font penser à une pédogenèse notable (faible taux de $\mathrm{CaCO}_{3}$, taux maximum de $\mathrm{C}$ et de $\mathrm{N}$ ) et, en même temps, le minimum de $\mathrm{Cl}^{-}$et, surtout, de $\mathrm{P}_{2} \mathrm{O}_{5}$.

L'incertitude sur l'âge de ce membre est due à l'écart, bien connu maintenant, relevé entre les datations au ${ }^{14} \mathrm{C}$ obtenues sur charbon de bois et celles obtenues sur coquilles provenant du même dépôt. Malgré cela, cet âge est tout à fait comparable à celui de la très basse terrasse historique reconnue, par exemple, en Tunisie et en Provence (Ballais, Crambes, 1992).

Comme plus à l'est, en Tunisie, en bordure de l'oued es Sgniffa ou en bordure de l'oued el Kseub, dans le même étage semi-aride, ce membre, déjà d'âge historique, vient se superposer aux dépôts antérieurs (Ballais, 1993), alors que, en général, en Tunisie, les dépôts d'âge historique sont emboîtés dans la basse terrasse holocène préhistorique (BallaIs, 1991a).

Alors que depuis la fin de l'accumulation du membre 2, le problème de l'intervention des sociétés humaines n'avait pu être posé, car aucune trace de leur présence n'existe dans la région, il en va différemment avec la période romaine et le début du Moyen Âge. Les Romains, en particulier, ont laissé de nombreuses marques de leur colonisation dans les Nemencha (Leveau, 1974-1975). Dans le détail, sur la coupe type, ils ont creuśé un canal d'irrigation dans le sommet du membre 4 où ils ont abandonné des tessons de céramique sigillée. Contemporains ou peu antérieurs sont les barrages entaillés dans ce membre 4 et suspendus, actuellement, de 1 à $4 \mathrm{~m}$ au-dessus du lit des oueds, ou encore presque intacts dans les cours amont. La colonisation romaine a donc, à la fois, affecté la végétation (forêt claire) et les sols, par le défrichement pour la mise en culture, mais aussi les cours d'eau par les travaux entrepris pour l'irrigation. Et cependant, ce n'est qu'en fin de période romaine, lors de l'Antiquité tardive, que l'accumulation reprend. Après une période de combinaison anthropo-climatique favorable (BallaIs, 1992c), cette accumulation reprend à la faveur d'un nouveau basculement des géosystèmes sous l'effet d'une petite pulsation climatique qui affecte plus l'intensité des précipitations que leur total. Cette pulsation ne devient efficace sur le plan morphogénique que grâce à la fragilisation des géosystèmes provoquée ici par plusieurs siècles de colonisation romaine, puisque les régions de l'étage saharien non colonisées n'ont pas été affectées (Ballais, 1994).

\subsection{La morphogenèse post-romaine :}

Alors que, depuis plus de 3000 ans, au minimum, la tendance générale était à l'accumulation, c'est l'entaille qui domine depuis la fin de l'Antiquité, une entaille vigoureuse, qui atteint près de $6 \mathrm{~m}$ à la coupe type, et une entaille rapide : en moyenne 3,5 a $4,2 \mathrm{~mm} / \mathrm{an}$, soit un peu plus vite que la moyenne du Maghreb oriental (Ballais, 1991c). En fait, les vitesses réelles ont été plus importantes puisqu'un bref renversement de tendance a permis l'accumulation de la très basse terrasse. Cette dernière pourrait être contemporaine de la très basse terrasse historique islamique, reconnue en Tunisie (BallaIs, 1991a, 1994), ou encore plus récente, compte-tenu de sa très faible altitude au-dessus du lit majeur. L'entaille est étroite, mais un lit majeur, limité, y existe, à la différence de l'entaille du Subboréal. Cependant, elle laisse subsister de larges témoins de la basse terrasse. 
Cette grande entaille d'âge historique est très générale autour de la Méditerranée (VrTa-Finz, 1969), mais il est difficile d'en connaître la cause. En effet, au Maghreb oriental, mais aussi en d'autres points du bassin méditerranéen, on a reconnu, au cours de l'Holocène, des phases d'accumulation contemporaines de celles décrites ici, mais chaque phase d'accumulation n'est pas systématiquement suivie par une phase d'entaille qui génèrerait ainsi une terrasse. En Tunisie, le nombre des terrasses semble d'ailleurs varier en fonction de l'étage bioclimatique considéré : on a recensé, jusqu'à présent, une seule terrasse dans l'étage saharien, deux (et rarement trois) dans l'étage aride, trois (et parfois deux) dans l'étage semi-aride, et une (et peut-être deux ou trois) dans l'étage subhumide. Par contre, l'entaille actuelle est très générale. En cohérence avec les hypothèses précédentes, elle doit correspondre à une fourniture de débris par les versants insuffisante pour saturer les écoulements. Une bonne couverture de sols et de végétation est alors favorable. Or, tout montre, surtout depuis plus d'un siècle, que la végétation est de plus en plus réduite, qu'elle couvre de moins en moins le sol : une steppe à Artemisia ou à Stipa tenacissima couvre les pentes sous un bioclimat favorable à la forêt de $P$ inus halepensis. Et les preuves d'érosion des sols sont spectaculaires. Il faut alors que les crues soient plus puissantes, ce que montre un certain nombre d'indices. En résumé, les cours d'eau, privés des réserves hydriques des sols et de leurs effets, sont devenus entièrement et immédiatement tributaires du régime pluvial ; alimentés par des averses intenses et relativement peu chargés, ils ont utilisé toute leur puissance à creuser. Les fluctuations climatiques historiques, en particulier le Petit Âge Glaciaire, ont pu jouer un rôle, mais aucun indice n'en existe aux environs de Chéria.

\section{CONCLUSION}

L'étude de l'évolution de la vallée de l'oued Chéria-Mezeraa confirme l'existence d'une morphogenèse tardiglaciaire caractérisée par l'accumulation de cônes alluviaux.

Pour la période holocène, l'absence de datations des dépôts les plus anciens, ne permet pas de reconnaître les étapes des schémas d'évolution paléoclimatiques proposés pour le Maghreb oriental (BALLAIs, 1992b). En revanche, l'Holocène moyen et supérieur est bien représenté et permet la mise en évidence d'une crise morphogénique contemporaine de celle de la rive septentrionale de la Méditerranée attribuée à l'Âge du Bronze et au début de l'Âge du Fer, ainsi que l'existence d'une période à bilan hydrique plus favorable que l'actuel vers $2400-2200$ B.P. L'Holocène se caractérise également par l'existence de trois phases d'entaille des cours d'eau : une, mal datée, avant l'optimum climatique, une seconde due à cette crise de l'Âge du Bronze-đébut de l'Age du Fer et la troisième, de beaucoup la plus importante, de l'époque médiévale à l'actuel. Cependant, elles ne dégagent que deux terrasses : la basse terrasse dont la genèse, fort complexe, s'étend sur plus de 4500 ans, soit d'avant 5830 B.P. jusqu'à 1350 B.P. au plus tôt, et la très basse terrasse.

Note technique : les analyses granulométriques, chimiques et minéralogiques ont été effectuées au Centre de Géomorphologie du C.N.R.S. à Caen, selon les protocoles habituels de ce laboratoire. Nous remercions Michèle Levant, Jacques Quillard et Michelle-Noelle Le Coustumer de leur collaboration.

\section{RÉFÉRENCES BIBLIOGRAPHIQUES}

Ballass J.-L., (1976). - Morphogenèse holocène dans la région de Chéria (Nemenchas, Algérie), Actes Symp. Versants en Pays Méditer., Aix-en-Provence, 1975, C.E.G.E.R.M., vol. V, p. 127-130.

Ballais J.-L., (1984). - Recherches géomorphologiques dans les Aurès (Algérie), A.N.R.T., Lille, 2 vol., 626 p.

Ballais J.-L., (1991a). - Les terrasses historiques de Tunisie, Z- Geomorph., Berlin, N.F., Suppl.-Bd. 83, p. 221 -226.

Ballass J.-L., (1991b). - Evolution holocène de la Tunisie saharienne et présaharienne, Méditerranée, Aix-en-Provence, 4, p. 31-38.

Ballais J.-L., (1991c). - Vitesses d'accumulation et d'entaille des terrasses alluviales holocènes et historiques au Maghreb oriental, Physio-Géo, Meudon, n²2/23, p. 89-94.

Ballais J.-L., (1992a). - Variations de l'environnement et industries préhistoriques au Pléistocène supérieur terminal au Maghreb oriental présaharien, Déserts - Passé, Présent, Futur, CTHS, Paris, p. 41-53.

Ballais J.-L., (1992b). - Morphogenèse et géosystèmes holocènes dans l'Atlas saharien oriental et le Bas-Sahara (Algérie, Tunisie), Würzb. Geogr. Arb., Würzburg, 84, p. 1-16.

Ballass J.-L., (1992c). - Le climat au Maghreb oriental : apports de la géomorphologie et de la géochimie, Coll. «Le climat à la fin de l'Âge du Fer et dans l'Antiquité (500 BC-500 AD), Méthodes d'approche et résultats», Les Nouvelles de l'Archéologie, Dijon, $\mathrm{n}^{\circ}$ 50, p. 27-31. 
Ballais J.-L., (1993). - Morphogenèse fluviatile holocène en Tunisie, Travaux U.R.A. 903 du CNRS, Aix-en-Provence, $\mathrm{n}^{\circ} \mathrm{XXII}$, p. 63-78.

BALLAIS J.-L., (1994). - Alluvial Holocene terraces in eastern Maghreb : climate and anthropogenic controls, In : J. Lewn, M.-G. MACKLn, J.C. WOODWARD eds Mediterranean Quaternary River Environments, Balkema, Rotterdam, sous presse.

Ballais J.-L., Crambes A., (1992). - Morphogenèse holocène, géosystèmes et anthropisation sur la Sainte-Victoire, Méditerranée, Aix-en-Provence, 1-2, p. 29-41.

Ballais J.-L., Jorda M., Provansal M., Covo J., (1993). - Morphogenèse holocène sur le périmètre des Alpilles, Travaux du Centre Camille Jullian, Aix-en-Provence, $n^{\circ} 14$, p. 515-547.

Brun A., (1985). - La couverture steppique en Tunisie au Quatemaire supérieur, C.R. Acad. Sc. Paris, t. 301, série II, n' 14, p. 1085-1090.

Coque R., (1962). - La Tunisie présaharienne. Etude géomorphologique, A. Colin, Paris, 476 p.

FarRand W.R., Stearns C.H., Jackson H.E., (1982). - Environmental setting of Capsian and related occupations in the high plains of Northeastern Algeria, Geol. Soc. of America, Abstracts with Program, vol. 14, $n^{\circ} 7$, p. 487.

Grebenart D., (1975). - Le Capsien des régions de Tébessa et d'Ouled Djellal, Algérie, Université de Provence, Aixen-Provence, $335 \mathrm{p}$.

Jorda M., Parron C., Provansal M, Roux M. (1993). - Etosion et détritisme holocène en Basse Provence calcaire. L'impact de l'anthropisation, Travaux du Centre Camille Jullian, Aix-en-Provence, n 14, p. $225-233$.

KAlla-DRIDI H., (1994). - Recherches géomorphologiques dans la plaine de Tébessa et ses bordures (Algérie orientale), Thèse Paris I, 290 p. + annexe, multig.

Leveau P., (1974-1975). - Une vallée agricole dans les Nemenchas dans l'Antiquité, B.C.T.H., Paris, n.s. 10-11, B, p. 102-121.

Lubell D., Ballais J.-L., Gautier A., Hassan F.A., (1975). - The Prehistoric cultural Ecology of Capsian Escargotiètes, Libyca, Alger, t. XX111, p. 43-121.

Lubell D., Hassan F.A., Gautigr A., Ballais J.-L., (1976). - The Capsian Escargotiètes, Science, Washington, vol. 191, p. $910-920$.

Lubell D., Gautier A., Leventhal E.T., Thompson M., Schwarcz H.P., Skinner M., (1982-83). - The Prehistoric cultural Ecology of Capsian Escargotières, Part II : Report on Investigations conducted during 1976 in tbe Bahiret Télidjène, Tébessa Wilaya, Algeria, Libyca, Alger, t. XXX-XXXI, p. 59-142.

Petit-Maire N., Burollet P.F., Ballais J.-L., Fontuone M., Rosso J.-C., LazaAr A., (1991). - Paléoclimats holocenes du Sahara septentrional. Dépôts lacustres et terrasses alluviales en bordure du Grand Erg oriental à l'extrême sud de la Tunisie, C.R. Acad. Sci. Paris, t. 312, série II, p. 1661-1666.

RITchIE J.C., (1984). - Analyse pollinique de sédiments holocènes supérieurs des hauts plateaux du Maghreb oriental, Pollens et Spores, Montpellier, vol. XXVI, n 3-4, p. 489-496.

Roubet C., (1979). - Economie pastorale préagricole en Algérie orientale. Le Néolithique de tradition capsienne. Exemple : l'Aurès, C.N.R.S., Paris, 595 p.

Vrta-Finzi C., (1969). - The Mediterranean Valleys. Geological Changes in Historical Times, Cambridge Univ. Press, $140 \mathrm{p}$. 